\title{
EL IMPACTO DE LA CRISIS ECONÓMICA EN LA SALUD MENTAL DE LA POBLACIÓN
}

\author{
JOSEP MOYA OLLÉ1, MARCOS ANTONIO CATALÁN VEGA², MANUEL DE ARMAS HERNÁNDEZ³, \\ MARÍA TERESA ANGUERA ARGILAGA ${ }^{4}$, ESTER FORNELLS ADMELLA ${ }^{5}$ \\ Y JAUME SATÓ GELI ${ }^{6}$ \\ ${ }^{1}$ Observatori de Salut Mental de Catalunya. Fundació Parc Taulí. Sabadell (Barcelona). \\ ${ }^{2}$ Corporació Sanitària Parc Taulí. Fundació Parc Taulí. Biblioteca. Sabadell (Barcelona). \\ ${ }^{3}$ Departamento de Educación. Secretario de la Facultad de Formación del Profesorado. \\ Universidad de Las Palmas de Gran Canaria. \\ ${ }^{4}$ Departament de Metodologia de les Ciències del Comportament. Institut de Recerca IR3C. \\ Facultat de Psicologia. Campus Mundet. Barcelona. \\ ${ }^{5}$ Consell Comarcal del Baix Llobregat. Sant Feliu de Llobregat (Barcelona). \\ ${ }^{6}$ Corporació Sanitària Parc Taulí. Sabadell (Barcelona).
}

\section{RESUMEN}

La crisis económica que se desencadenó a partir del año 2008 a nivel mundial está provocando efectos devastadores en la salud de la población, especialmente en la salud mental. Ello se traduce en un aumento de las consultas en los dispositivos de atención primaria, de salud mental y de servicios sociales básicos. En este artículo se expone un estudio cualitativo, descriptivo, de carácter exploratorio y fenomenológico con análisis de contenido, en el que se confirma la hipótesis exploratoria planteada, ya que, en efecto, la situación de crisis económica, que se concreta en forma de pérdida del puesto de trabajo, tiene unos efectos adversos sobre la salud mental de las personas afectadas. Finalmente se expone sucintamente el proyecto de lucha contra la pobreza y el riesgo de exclusión social llevado a cabo con los grupos de dinamización social.

Palabras clave: crisis económica, salud mental, grupos de dinamización social.

Correspondencia: J. Moya

Correo electrónico: jmoya@tauli.cat 


\section{INTRODUCCIÓN}

La actual crisis económica está provocando efectos muy negativos en la salud de la población, especialmente en lo que atañe a la salud mental. Esto se traduce en un aumento de las consultas en los dispositivos asistenciales de atención primaria, básicamente por problemas de ansiedad y depresión ${ }^{1}$. Estas consultas presentan unos contenidos discursivos diferentes según las franjas de edad. Así, cuando se trata de población adolescente o joven, los pacientes hablan de falta de expectativas de futuro y de la inutilidad de sus esfuerzos académicos, ya que el mercado de trabajo no les proporciona las ofertas adecuadas a su preparación y formación. Cuando se trata de personas que estaban insertadas en el mundo laboral, las narrativas se refieren a pérdida del puesto de trabajo y de las enormes dificultades para poder acceder a un nuevo empleo.

Karanikolos et al. ${ }^{2}$ nos indican que España, Grecia y Portugal son los países con mayor afectación de la salud mental en relación con la crisis económica, y enfatizan la idea de que la recesión económica tiene efectos sobre la salud, concluyéndose que la interacción entre austeridad fiscal, shock económico y débil protección social aumenta dicho riesgo.

Por otro lado, Cortés-Franch y González López Valcárcel ${ }^{3}$ nos dicen que hay evidencia de la relación de la crisis con la salud de la población española, con las desigualdades en salud, con los cambios en algunos estilos de vida y con el acceso a servicios sanitarios. La crisis ha impactado en muchos determinantes estructurales de la salud, y se identifican distintos grupos de población que son más vulnerables a los efectos de la crisis. Generalmente, las respuestas políticas sobre cómo gestionar las crisis no han tenido en cuenta la evidencia existente. La crisis puede facilitar la vulnerabilidad de las políticas públicas a la acción de las corporaciones, poniendo en riesgo la implantación de políticas saludables, sobre todo en contextos de reducción del papel y el tamaño de los estados para mejorar la economía.
Borrell, Rodríguez-Sanz, Bartoll, Malmusi y Novoa ${ }^{4}$ consideran que para estudiar el impacto de la crisis en la salud de la población es necesario: a) analizar los determinantes de la salud y otros indicadores de salud además de la mortalidad; $b$ ) tener en cuenta los diferentes ejes de desigualdad y, sobre todo, a los grupos más desfavorecidos, y $c$ ) seguir controlando y estudiando los efectos a más largo plazo, ya que la situación socioeconómica de nuestro país tardará mucho en recuperar los niveles de hace años.

También queremos destacar las aportaciones de Chang et al. ${ }^{5}$ que investigan el impacto de la crisis económica mundial de 2008 sobre las tendencias internacionales en el suicidio, para identificar el sexo/grupos de edad y los países más afectados. Los resultados de dicha investigación mostraron un aumento de suicidios entre los hombres en 27 países europeos y en 18 países de América, donde las tasas de suicidio eran del $4,2 \%(3,4 \%$ a $5,1 \%)$ y del $6,4 \%$ (5,4\% a 7,5\%), superior en el 2009 de lo esperado si la crisis del 2008 no hubiera aparecido. No obstante, para las mujeres no hubo ningún cambio en los países europeos y el aumento del suicidio en América fue menor que en los hombres (2,3\%). El aumento de los suicidios en los hombres europeos fue más elevado en personas de 15-24 años (11,7\%), mientras que en los países de América los hombres de 45-64 años de edad mostraron el mayor incremento (5,2\%). El aumento de las tasas de suicidio en los hombres parecía estar asociada con el incremento del desempleo, especialmente en los países con bajos niveles de desempleo antes de la crisis. En definitiva, después de la crisis económica de 2008, las tasas de suicidio aumentaron en los países europeos y americanos estudiados, sobre todo entre los hombres y en los países con mayores niveles de pérdida de empleo.

Para las mujeres, no hubo ningún cambio en los países europeos y el aumento en las Américas fue menor que en los hombres (2,3\%). El aumento de los suicidios en los hombres europeos fue mayor en 
personas de 15 a 24 años (11,7\%), mientras que en los países de América mostraron mayor incremento los hombres de 45 a 64 años (5,2\%). El aumento de las tasas de suicidio en los hombres parecía estar asociada con la magnitud de aumento del desempleo, especialmente en los países con bajos niveles de desempleo antes de la crisis. En definitiva, después de la crisis económica de 2008, las tasas de suicidio aumentaron en los países europeos y americanos estudiados, sobre todo en los hombres y en los países con mayores niveles de pérdida de empleo.

Un factor de gran peso específico en cuanto a sus efectos sobre la salud es el que se refiere a las desigualdades sociales. Este factor ha sido señalado por varios autores ${ }^{6-8}$; así, el primero, Wilkinson, advirtió que en la actualidad queda claro que los niveles de salud de la población se ven totalmente afectados no tanto por las atenciones médicas como por las circunstancias sociales y económicas en las que la gente vive y trabaja ${ }^{6}$. El mismo autor argumentaba que algunas de las relaciones más importantes entre nuestra salud y las condiciones de vida son las relaciones psicosociales, es decir, que muchos de los procesos biológicos que conducen a la enfermedad se desencadenan por lo que pensamos y sentimos respecto de nuestras circunstancias sociales y materiales.

Las personas que tienen un determinado nivel de renta, especialmente las de las rentas más bajas, parecen estar más sanas en lugares igualitarios. Esto quiere decir que a mayor igualdad encontramos mejor salud. Wilkinson establece la hipótesis de que las personas suelen confiar más unas en otras en los lugares en los que las diferencias de renta son menores.

Estatus social, amistad y cohesión social son tres factores que ejercen una influencia considerable en la salud. Wilkinson se preguntaba por qué era tan importante la amistad, por qué las diferencias en el estatus social podían tener un efecto tan grande en la salud. Y, en un intento de responder a la pregun- ta, afirmaba que «los niveles de salud de la población en el mundo desarrollado están dominados cada vez más por una gama de variables psicosociales que resultan muy importantes para la salud». Entre ellas destaca el control que realmente poseen las personas sobre el trabajo y sobre otras facetas de la vida; el desequilibrio percibido entre los esfuerzos emocionales y las recompensas planteadas y obtenidas de su trabajo; el tener que hacer frente a sucesos vitales estresantes o a dificultades constantes; los vínculos débiles y dificultades emocionales a una edad temprana, y, finalmente, las relaciones sociales negativas.

En un estudio posterior, Wilkinson y Piket ${ }^{7}$ se plantearon la pregunta sobre qué diferencias suponen las desigualdades de renta en los países del Primer Mundo.

Para averiguarlo, recogieron datos comparables internacionalmente tanto sobre salud como de otros problemas sociales. Los problemas sobre los cuales obtuvieron datos fueron: nivel de confianza, trastornos mentales, esperanza de vida y mortalidad, obesidad, madres adolescentes, rendimiento escolar, homicidios, tasas de población reclusa y, finalmente, movilidad social. Los autores concluyeron que los problemas sociales y de salud tienden claramente a ser menos frecuentes en los países más igualitarios. Cuanto mayor es la desigualdad, mayor es la puntuación dentro del índice de problemas sociales y de salud.

Otro punto que es interesante destacar es el que se refiere a los efectos cognitivos de la pobreza. Stiglitz ${ }^{8}$ señaló que el estrés provocado por no tener suficiente dinero para hacer frente a las necesidades urgentes puede afectar a la capacidad de tomar decisiones que podrían contribuir a aliviar la situación. En este sentido, las limitadas reservas de recursos cognitivos se agotan y ello puede llevar a las personas a tomar decisiones fatídicas.

A partir de estas consideraciones así como de la recogida sistemática de los relatos de los pacientes que acudían a los dispositivos de salud mental por 
problemas derivados de los efectos de la crisis económica nos propusimos, desde el Observatorio de Salud Mental de Cataluña, realizar un estudio exploratorio con un doble enfoque: cualitativo y cuantitativo, con el objetivo de captar la realidad de personas que acudían a los centros de salud refiriendo todo un conjunto de malestares emocionales, así como las estrategias que desarrollan para enfrentarse a ellos.

\section{OBJETIVOS}

A partir de la realidad actual, los objetivos que han impulsado el presente estudio han sido los siguientes:

1. Recoger los relatos, las quejas y los malestares de las personas que acuden a los servicios sociales básicos por causas directamente relacionadas con la crisis económica.

2. Conocer cuáles son las estrategias que estos usuarios ponen en marcha para enfrentarse (o no) a una situación que aparece de forma brusca y que afecta a sus vidas.

3. Profundizar en las actitudes y motivaciones de estas personas frente a la situación que viven.

4. Conocer las opiniones de los profesionales de los servicios sociales de Atención Primaria hacia las problemáticas derivadas directamente de la crisis económica que explican los usuarios de sus servicios.

5. Conocer las opiniones de los profesionales de los centros de salud mental de adultos hacia las problemáticas derivadas directamente de la crisis económica que explican los pacientes atendidos en sus dispositivos.

\section{HIPÓTESIS}

La hipótesis exploratoria que se plantea consiste en comprobar si la situación de crisis económica, que se concreta en forma de pérdida del puesto de tra- bajo, tiene unos efectos adversos sobre la salud mental de las personas afectadas.

\section{MÉTODO}

Se siguió una metodología cualitativa, ya que lo que se pretendió fue profundizar en la dimensión psicológica y psicosocial del usuario, comprender la problemática que vive, cómo le afecta el problema y los mecanismos que pone en marcha para hacerle frente. Se analizaron las percepciones, las actitudes, las motivaciones y las emociones a través del discurso del usuario.

El trabajo de campo se desarrolló en dos fases. La primera, centrada en la realización de grupos de discusión de profesionales de servicios sociales básicos y de centros de salud mental, se llevó a cabo entre diciembre de 2011 y octubre del 2012. La segunda, centrada en grupos de discusión de usuarios de servicios sociales básicos, se desarrolló entre noviembre de 2012 y noviembre de 2013.

\section{Participantes}

Los participantes de la primera fase fueron ocho grupos de discusión de profesionales de los centros de salud mental y de servicios sociales básicos de la ciudad de Barcelona (concretamente, el Distrito de l'Eixample) y tres municipios de su conurbación (Martorell, Sabadell y Sant Feliu de Llobregat).

En los grupos de discusión de profesionales (formados por un total de 88 personas) participaron profesionales de las áreas de psiquiatría (22,72\%), psicología (13,63\%), trabajo social (42,04\%), enfermería $(4,54 \%)$ y educación social $(17,04 \%)$.

Los participantes de la segunda fase fueron usuarios de servicios sociales básicos que habían acudido a estos dispositivos por presentar problemas causados directamente por la situación de desempleo y/o desahucio. Se constituyeron siete grupos de discusión, aunque inicialmente se habían previsto ocho, dos por cada sector de población. Un grupo de usua- 
rios no pudo realizarse debido a que el número de personas que acudió a la convocatoria fue insuficiente.

\section{Material}

Para proceder a la obtención de datos cualitativos se preparó una batería de preguntas para los participantes de cada grupo de discusión.

Las preguntas planteadas a los profesionales de centros de salud mental de adultos se centraron en conocer si habían detectado un incremento de las consultas motivadas por problemas derivados directamente de la crisis económica, así como las modalidades de malestares y síntomas predominantes. En cuanto a los profesionales de servicios sociales básicos, también se les preguntó si habían constatado un incremento de las demandas motivadas por problemas relacionados con la crisis económica y sobre las estrategias que utilizaban los usuarios para hacer frente a las penurias económicas.

Finalmente, las preguntas que se plantearon a los usuarios de los servicios sociales básicos se centraron en conocer cómo había repercutido en ellos el hecho de haber perdido el puesto de trabajo, cómo había afectado a sus relaciones familiares y/o sociales y cuál había sido el mejor apoyo que habían encontrado.

El software utilizado para la codificación de las transcripciones correspondientes a los grupos focales de profesionales fue ATLAS.ti (v5). Posteriormente, para la codificación de los grupos focales de usuarios de servicios sociales básicos se utilizó el ATLAS.ti (v7.1.6), escogido por sus mejores prestaciones en el análisis de material textual y por las posibilidades ofrecidas para un tratamiento posterior de la información ${ }^{9}$.

\section{Procedimiento}

Para poder realizar el estudio sobre los malestares vinculados a los problemas relacionados con la cri- sis económica se recurrió a los grupos de discusión ${ }^{10-12}$. Estos grupos de discusión consisten en conversaciones planeadas y que están diseñadas para obtener información de un área definida de interés; en este caso, qué tipo de malestares y síntomas se generan en las personas afectadas por la crisis económica.

Los grupos de discusión se realizaron con un número aproximado de 7 a 10 personas, guiadas por un moderador experto. Los participantes en el grupo expusieron sus ideas y comentarios en común. Es un procedimiento muy adecuado cuando se trata de explicar cómo perciben las personas una determinada experiencia, y que hoy en día está siendo utilizado de forma creciente en ámbitos de salud mental ${ }^{13-16}$.

Como se ha señalado, el estudio se desarrolló en dos fases:

Fase 1: centrada en los profesionales de los servicios sociales y en los profesionales del ámbito de la salud mental que dan apoyo directo a las áreas básicas de salud y, por tanto, podían recoger los testimonios de los profesionales (medicina de familia, enfermería y trabajo social) así como de aquellos usuarios que acuden presentando malestares y problemas de salud desencadenados por la situación de precariedad social.

Fase 2: centrada en los usuarios de los servicios sociales básicos atendidos durante el primer trimestre del año 2012 por presentar problemáticas directamente relacionadas con la crisis económica y que constan en las memorias de los equipos de Servicios Sociales en el apartado "problemas económicos y laborales».

Los participantes se distribuyeron de la siguiente forma:

Fase 1: funcionaron 8 grupos de discusión ( 4 del área de los Servicios Sociales y 4 del área de la salud mental, diferenciados por poblaciones: Barcelona, Martorell, Sabadell y Sant Feliu de Llobregat). 
Fase 2: Se plantearon 7 grupos de discusión constituidos por usuarios de servicios sociales básicos que cumplían los siguientes criterios:

- Personas atendidas en servicios sociales básicos en el período enero-abril de 2012.

- Personas que habían acudido por problemas directamente relacionados con la crisis económica.

- Personas no atendidas en los dispositivos específicos de salud mental.

- Personas que no presentaran trastornos mentales graves (esquizofrenia, trastorno bipolar, etc.) aunque sí podían presentar síntomas leves como insomnio, tristeza o estados de ansiedad.

Para analizar los contenidos de las conversaciones se elaboró un sistema de categorías para cada pregunta, cumpliendo los requisitos de exhaustividad y mutua exclusividad.

\section{RESULTADOS}

Los datos se segmentaron por grupos de informantes y categorías, se analizaron cualitativamente los contenidos de la información recogida de los diferentes grupos de discusión, identificando fragmentos significativos para determinar los fenómenos reflejados y su significado dentro del texto, y se estableció un código descriptivo para cada segmento del texto señalado. Las categorías elaboradas se generaron de forma mixta a partir de las guías preparadas con antelación y de los temas emergentes de los grupos de discusión. Nos referiremos únicamente a los resultados obtenidos en los grupos de discusión de los usuarios de servicios sociales básicos.

1. Sobre las repercusiones generales derivadas de haber perdido el puesto de trabajo, se obtuvieron las siguientes respuestas (tabla 1 ):

- En primer lugar: la inutilidad de las acciones emprendidas, con un $40,00 \%$.

- En segundo lugar: dependencias de otros, con un $28,00 \%$

- En tercer lugar: interrogarse sobre qué hacer, con un $12,00 \%$.

2. Sobre las repercusiones personales que ha tenido esta situación se han obtenido los resultados que

Tabla 1. Resultados a la pregunta 1 . ¿Cómo ha repercutido socialmente en ustedes el hecho de quedarse sin trabajo?

\begin{tabular}{|l|c|c|}
\hline \multicolumn{2}{|c|}{ socialmente en ustedes el hecho de quedarse sin trabajo? } & \\
\cline { 2 - 3 } Categorías & Frecuencia & Porcentaje \\
\hline Cambio considerable en las condiciones de vida & 5 & $10 \%$ \\
\hline Dependencia de los otros & 14 & $28 \%$ \\
\hline Interrogarse sobre qué hacer & 6 & $12 \%$ \\
\hline Inutilidad de la formación & 2 & $4 \%$ \\
\hline Inutilidad de acciones emprendidas & 20 & $40 \%$ \\
\hline Necesidad de abandonar la vivienda habitual & 1 & $2 \%$ \\
\hline Necesidad de consultar a un especialista en psiquiatría & 2 & $4 \%$ \\
\hline Totales & 50 & $100 \%$ \\
\hline
\end{tabular}




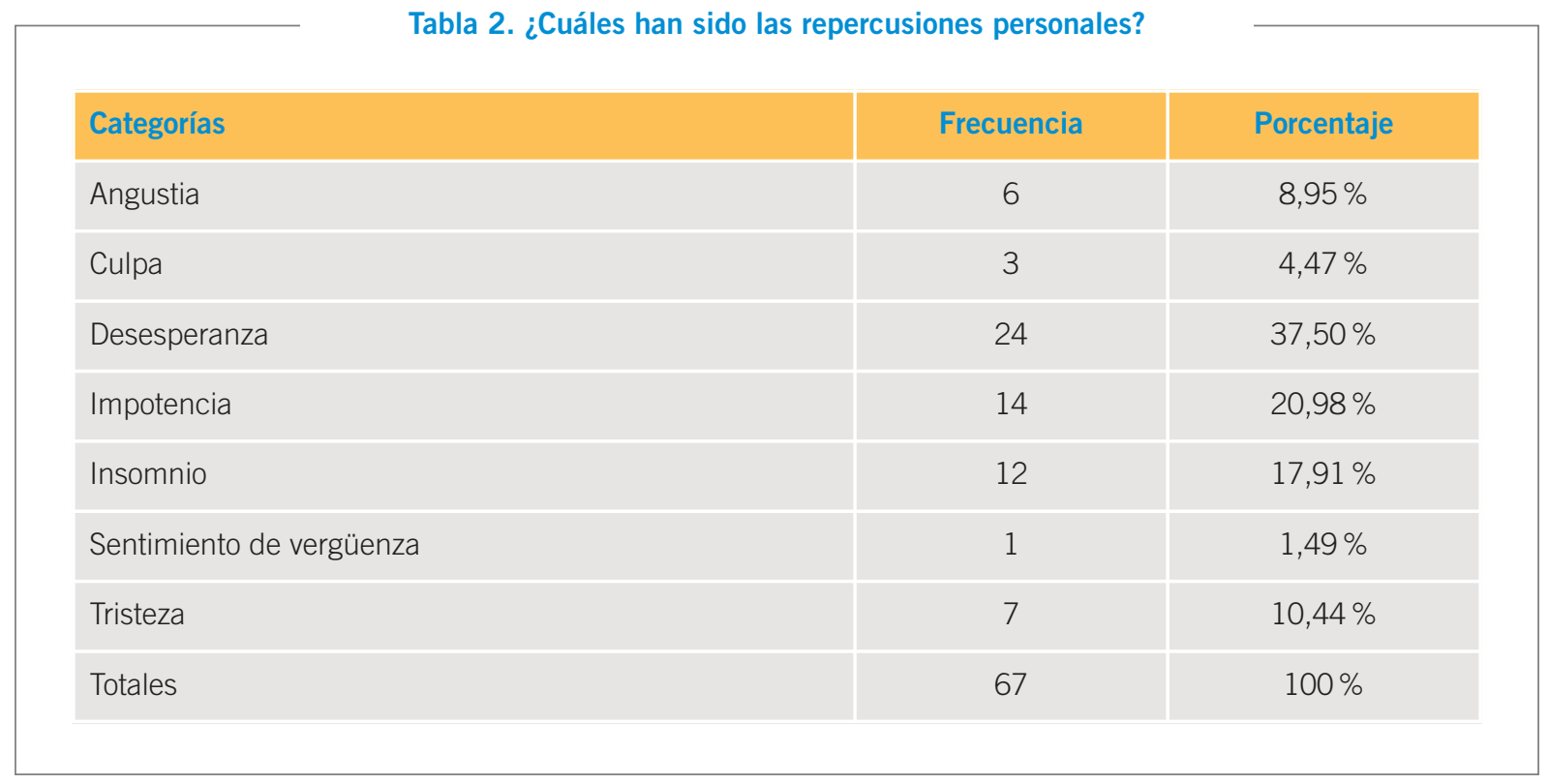

se muestran en la tabla 2. De esta tabla se desprende que el malestar o los síntomas referidos por los usuarios fueron:

- En primer lugar: desesperanza, con una frecuencia de 24 y que equivale al 37,50\%.

- En segundo lugar: impotencia, según opinión de los usuarios, con una frecuencia de 14 y que equivale al $20,89 \%$.

- En tercer lugar: insomnio, con una frecuencia de 12 y que equivale al $17,91 \%$.
3. Sobre cómo ha repercutido esta situación en sus relaciones familiares y sociales se han obtenido los resultados que se muestran en la tabla 3. De esta tabla se desprende que las principales repercusiones en sus relaciones fueron:

- En primer lugar: buen apoyo familiar, con una frecuencia de 15 y que equivale al 28,84\%.

- En segundo lugar: buen apoyo de los amigos, según opinión de los usuarios, con una frecuencia de 15 y que equivale al $28,84 \%$.

Tabla 3. ¿Cuáles han sido las repercusiones en sus relaciones familiares, de amigos, de círculo social?

\begin{tabular}{|l|c|c|}
\hline \multicolumn{2}{|c|}{ familiares, de amigos, de círculo social? } \\
\hline Categorías & Frecuencia & Porcentaje \\
\hline Ausencia de apoyo de los amigos & 10 & $19,23 \%$ \\
\hline Ausencia de apoyo familiar & 12 & $23,03 \%$ \\
\hline Buen apoyo de los amigos & 15 & $28,84 \%$ \\
\hline Buen apoyo familiar & 15 & $28,84 \%$ \\
\hline Totales & 52 & $100 \%$ \\
\hline
\end{tabular}


Tabla 4. ¿Qué han hecho ustedes frente a esta situación?

\begin{tabular}{|l|c|c|}
\hline \multicolumn{1}{|l|}{ Tabla 4. ¿Qué han hecho ustedes frente a esta situación? } & \multicolumn{2}{c|}{ Porcentaje } \\
\hline Categorías & Frecuencia & $48,01 \%$ \\
\hline Estudiar y mejorar la formación & 12 & $4,01 \%$ \\
\hline Imaginarse en un punto extremo & 1 & $24,01 \%$ \\
\hline Pensar que algún día mejorará la situación & 6 & $24,01 \%$ \\
\hline Pensar que Dios o algún ente superior intervendrá en su favor & 6 & $100 \%$ \\
\hline Totales & 25 & \\
\hline
\end{tabular}

- En tercer lugar: ausencia de apoyo familiar, con una frecuencia de 12 y que equivale al 23,03\%.

4. Sobre qué tipo de acciones habían tomado los usuarios afectados, se obtuvieron los resultados que se muestran en la tabla 4. De esta tabla se desprende que las acciones emprendidas son:

- En primer lugar: estudiar y mejorar la formación, con una frecuencia de 12 y que equivale al $48,01 \%$.

- En segundo lugar: pensar que algún día mejorará la situación, según opinión de los usuarios, con una frecuencia de 6 y que equivale al 24,01\%.
- En tercer lugar: pensar que Dios o algún ente superior intervendrá en su favor, con una frecuencia de 6 y que equivale al 24,01\%.

5. Sobre cuál ha sido el mejor apoyo que han encontrado, se han obtenido las respuestas que se detallan en la tabla 5. De esta tabla se desprende que el malestar o los síntomas fueron:

- En primer lugar: la familia fue el mejor apoyo, con una frecuencia de 21 y que equivale al $48,83 \%$.

- En segundo lugar: los servicios sociales fueron el mejor apoyo, según opinión de los usuarios,

Tabla 5. ¿Cuál es el mejor apoyo que han encontrado ustedes?

\begin{tabular}{|l|c|c|}
\hline Categorías & Frecuencia & Porcentaje \\
\hline El mejor apoyo es la fe, las creencias religiosas & 3 & $6,97 \%$ \\
\hline $\begin{array}{l}\text { El mejor apoyo procede de las personas que he conocido } \\
\text { en esta misma situación }\end{array}$ & 1 & $2,32 \%$ \\
\hline El mejor apoyo son los amigos & 6 & $14,01 \%$ \\
\hline El mejor apoyo es la familia & 21 & $48,83 \%$ \\
\hline El mejor apoyo han sido los servicios sociales & 11 & $25,58 \%$ \\
\hline Tomar la situación con ironía o humor & 1 & $2,32 \%$ \\
\hline Totales & 43 & $100 \%$ \\
\hline
\end{tabular}


con una frecuencia de 11 y que equivale al $25,58 \%$.

- En tercer lugar: el mejor apoyo han sido los amigos, con una frecuencia de 6 y que equivale al $14,01 \%$.

\section{DISCUSIÓN Y CONCLUSIONES}

Los resultados descriptivos obtenidos nos aportan una respuesta respecto a la hipótesis exploratoria y permitieron cumplir los objetivos propuestos. El incremento observado de las demandas de visitas coincidió con diversos estudios ${ }^{17-20}$ y alertó sobre los efectos adversos de esta crisis sobre la salud mental de la población.

Sin embargo, para tener una visión más precisa de cómo se produce este impacto es necesario tener en cuenta que todo ello acontece en un marco social caracterizado por la llamada sociedad del hiperconsumo ${ }^{21}$. Lipovetsky explicó que en esta sociedad, el individuo se ve impelido a construir de modo individualizado el propio estilo de vida y el empleo del tiempo a acelerar las operaciones de la vida corriente, a aumentar la capacidad de relacionarse y a corregir las imperfecciones del cuerpo. Sin embargo, al romper las últimas costumbres y tradiciones estructurantes, la revolución del consumo ha dejado a los individuos a merced de sus propias fuerzas, y estos deben afrontar los problemas de la existencia sin contar con regulaciones ni apoyos colectivos. Es así como, reducido a sí mismo, el individuo no está ya preparado para soportar los infortunios de la existencia. Además, sometido a un diluvio de incitaciones a gozar de la vida, ve cómo aumenta inevitablemente la distancia entre las promesas del paraíso y lo real.

En este contexto de crisis económica, el aumento de la decepción de las personas no deriva únicamente de los despidos, sino que arraiga igualmente en los ideales individualistas de plenitud personal, vehiculados a gran escala por la sociedad del hiperconsumo ${ }^{22}$.
Las personas acuden a los servicios sociales básicos y a las áreas básicas de salud manifestando predominantemente el sentimiento de que han perdido el control sobre sus propias vidas. Se trata de un sentimiento de impotencia y desbordamiento, de no saber qué hacer para reconducir la situación. En esta misma línea se sitúan las categorías de «bloqueo» e «inhibición», que afectan a muchas personas y les impiden llevar a cabo las acciones necesarias para intentar salir de la situación adversa. En un estudio publicado unos meses antes de que estaIlara la crisis, el sociólogo Sennett ${ }^{23}$ se encontró con unos colectivos pertenecientes a la clase media que se hallaban dominados por la sensación de que su vida había quedado a la deriva.

En ocasiones, el sentimiento de impotencia puede convertirse en desesperanza con el correlato dramático del acto suicida. Cabe destacar que los profesionales de salud mental refirieron que muchos pacientes y/o usuarios manifestaron ideas de suicidio. No obstante, no se trata tanto de un deseo de poner fin a la propia vida como de poner un límite -absoluto- a un sufrimiento para el que no se encuentra ninguna salida. En consecuencia, no solo hay que tener presente la posibilidad del acto suicida, sino también el hecho de que la ideación autolítica se convierta en el vector principal en la vida de estas personas.

Por otro lado, las personas afectadas por la situación de paro laboral explicaron diversos tipos de acciones o estrategias. La más referida fue incrementar el nivel de formación profesional, en un claro intento de ampliar el posible campo de reinserción laboral. Pero, además, emergió una segunda estrategia consistente en pensar que las cosas podrán mejorar algún día o bien que Dios o algún ente superior intervendrá en su favor y de este modo podrán encontrar un nuevo trabajo. Sin embargo, esa actitud de esperar que una solución mágica les permita encontrar la solución a sus problemas económicos puede ser la antesala de una caída depresiva, y es precisamente en este punto en el que muchos profesio- 
nales consideran que se debería llevar a cabo una estrategia preventiva orientada a la comunidad ${ }^{24}$.

Ello se justifica sobre la base de que es en el seno de la comunidad donde se producen los distintos hechos que llevan al crecimiento de los sujetos, donde se generan las dificultades y donde se producen las situaciones necesarias para hacerles frente ${ }^{25}$. En este marco, el trastorno mental es el fracaso de la relación de un sujeto con su medio y tiene siempre serias repercusiones en las modalidades de los vínculos. La angustia, la depresión o el acto suicida son manifestaciones dramáticas de aquel fracaso, y es indudable que ello requiere de todo un conjunto de intervenciones individuales (psicoterapia, tratamiento psicofarmacológico, trabajo social), pero también precisa de una incidencia en el ámbito comunitario, pues solo así se podrá ejercer una acción de prevención.

Finalmente, los usuarios de servicios sociales consideraron que el mejor apoyo lo han encontrado en la familia, los amigos y/o los propios servicios sociales; aunque cabe destacar también que en bastantes casos, la categoría «ausencia de apoyo familiar» presenta una frecuencia destacable. Estos últimos resultados pueden ser la manifestación de que aunque nos encontramos inmersos en una sociedad considerablemente individualista, persisten todavía los vínculos de apoyo tanto en el seno de las familias como en los círculos de amistades. Una dirección que los profesionales podríamos potenciar. Ha sido precisamente este el marco que nos ha movido a promover los grupos de dinamización social, cuyo objetivo principal es prevenir y evitar la exclusión social de las personas afectadas por la crisis económica actual, mejorando sus condiciones psicosociales y de salud mental y potenciando sus competencias e iniciativas en los ámbitos económico, social y familiar con los consecuentes efectos preventivos que ello tendrá en su nivel de salud integral. El Observatorio de Salud Mental de Cataluña, junto con el Consell Comarcal del Baix Llobregat y el equipo CIPAIS, de Barcelona, hemos iniciado un proyecto, cofinanciado por la Obra Social de la Caixa, que consta de tres pilares. Un primer pilar, constituido por la formación ad hoc de profesionales de servicios sociales básicos; un segundo pilar, constituido por los propios grupos de dinamización social, integrados por personas que se encuentran en situación de paro prolongado. Finalmente, un tercer pilar centrado en la elaboración de una Plataforma Digital, cuya finalidad es la de fomentar y facilitar los vínculos sociales entre los participantes de los grupos de dinamización social.

La experiencia acumulada hasta ahora avala las expectativas depositadas en el proyecto y constituye un acicate para generalizar esta metodología.

\section{BIBLIOGRAFÍA}

1. Gili M, Roca M, Basu S, McKee M, Stuckler D. The mental health risks of economic crisis in Spain: evidence from primary care centres, 2006 and 2010. Eur J Public Health. 2013;23(1):103-8.

2. Karanikolos M, Mladovsky P, Cylus J, Thomson S, Basu S, Stuckler D, et al. Financial crisis, austerity, and health in Europe. Lancet. 2013;381(9874):1323-31.

3. Cortès-Franch I, González López-Valcárcel B. Crisis económico-financiera y salud en España. Evidencia y perspectivas. Informe SESPAS 2014. Gac Sanit. 2014; 28 Suppl 1:1-6.

4. Borrell C, Rodríguez-Sanz M, Bartoll X, Malmusi D, Novoa AM. El sufrimiento de la población en la crisis económica del Estado español. Salud Colectiva. 2014;10(1)-95-8.

5. Chang SS, Stuckler D, Yip P, Gunnell D. Impact of 2008 global economic crisis on suicide: time trend study in 54 countries. BMJ. 2013;347:f5239.

6. Wilkinson R. Las desigualdades perjudican. Jerarquía, salud y evolución humana. Barcelona: Crítica; 2001.

7. Wilkinson R, Pickett K. Desigualdad. Un análisis de la (in)felicidad colectiva. Madrid: Turner; 2009.

8. Stiglitz J. El precio de la desigualdad. Madrid: Taurus; 2012.

9. Anguera MT. Tratamiento cualitativo de datos. En: Anguera MT, Arnau J, Ato M, Martínez MR, Pascual J, Vallejo G, editores. Métodos de investigación en Psicología. Madrid: Síntesis, 1995. p. 549-76.

10. Krueger R. Grupos de discusión. Madrid: Pirámide; 1988. 
11. Suárez M. El grupo de discusión. Una herramienta para la investigación cualitativa. Barcelona: Laertes; 2005.

12. Gutiérrez J. Dinámica del grupo de discusión. Madrid: Centro de Investigaciones Sociológicas; 2008.

13. Kadam UT, Croft P, McLeod J, Hutchinson M. A qualitative study of patients' views on anxiety and depression. Br J Gen Pract. 2001;51(466):375-80.

14. Nicholson J, Albert K, Gershenson B, Williams V, Biebel K. Family options for parents with mental illnesses: a developmental, mixed methods pilot study. Psychiatr Rehabil J. 2009;33(2):106-14.

15. Saavedra J, Cubero M, Crawford P. Incomprehensibility in the narratives of individuals with a diagnosis of schizophrenia. Qual Health Res. 2009;19(11):1548-58.

16. Wegner W, Pedro EN. Health conceptions under the perspective of lay caregiver women accompanying hospitalized children. Rev Lat Am Enfermagem. 2009; 17(1):88-93.

17. Álvaro JL, Fraser C. The psychological effects of unemployment in Spain. Int J Sociol Soc Policy. 1994;14(9): 1-19.

18. Buendía J. El impacto psicológico del desempleo. Murcia. Editum; 2010.
19. Boatas F, Hospital D, Surribas E, Serrano S. Factores desencadenantes de trastornos adaptativos: impacto de la crisis socioeconómica. XV Congreso Nacional de Psiquiatría (ponencia P0-457). Oviedo, 8 a 11 de noviembre de 2011.

20. Willmott G. Mental health in times of economic crisis. Brussels: European Parlament. Directorate-General for Internal Policies; 2012.

21. Lipovetsky G. La felicidad paradójica. Barcelona: Anagrama; 2007.

22. Lipovetsky G. La sociedad de la decepción. Barcelona: Anagrama; 2008.

23. Sennet R. La cultura del nuevo capitalismo. Barcelona: Anagrama; 2006.

24. Thornicrotf G, Tansella M. La matriz de la salud mental: manual para la mejora de servicios. Madrid: Triacastela; 2005.

25. Leal J. La relación en los cuidados y el trabajo en red en salud mental. En: Leal J, Escudero A, coordinadores. La continuidad de cuidados y el trabajo en red en salud mental. Madrid: Asociación Española de Neuropsiquiatría; 2006. p 29-57. 